\title{
Intracaval and Intracardiac Extension of Malignant Thymoma
}

\author{
Sachiko Futami, Tomio Yamasaki, Rieko Minami, Kohji Matsuba, Keisuke Inoue, Shigeki Nishimoto, \\ Shinichirou Matsumoto, Tatsurou AsadA*, Kyoichi Ogawa* and Masaru Furumoto**
}

A case of malignant thymoma presenting as the superior vena cava syndrome is reported. A 56-year old male was admitted with superior vena cava syndrome. CT and NMR-CT scan showed a solid homogenous superior mediastinal mass; which filled the lumen of the superior vena cava and the right atrium. A biopsy of the right atrial mass showed myxoma, and operation was performed. Histopathologically the tumor was diagnosed as thymoma. Intracaval and intracardiac extension of a thymoma is very rare.

(Internal Medicine 32: 257-260, 1993)

Key words: superior vena cava syndrome, mediastinal tumor

\section{Introduction}

Common causes of superior vena cava syndrome are obstruction or direct invasion into superior vena cava by neoplasm, and compression by metastatic lymph nodes. We report a case with superior vena cava syndrome which demonstrates intravascular extension of malignant thymoma.

\section{Case Report}

A 56-year-old man was admitted to the hospital because of face and upper extremity edema. The patient was in good health until two months earlier, when swelling and pain in his neck developed. One month before admission marked respiratory distress developed. Examination revealed engorged neck veins and marked edema in the upper trunk and extremities. No superficial lymphadenopathy was found. The lungs were clear. The heart was normal; no pericardial friction rub, or murmur was heard. The liver was palpable $2 \mathrm{~cm}$ below the costal arch in the right mid clavicular line, and the surface was smooth. There was no ascites.

Initial laboratory data showed a hematocrit value of $42.8 \%$; the white-cell count was 5100 , with $66 \%$ neutrophils. Blood chemistry findings were all within normal limits. Arterial blood gases showed $\mathrm{pO}_{2}$ of $55 \mathrm{mmHg}$, $\mathrm{pCO}_{2}$ of $31 \mathrm{mmHg}$ and $\mathrm{pH}$ of 7.47. Serological tumor markers were negative.

An electrocardiogram taken on admission revealed a normal rhythm at a rate of 86 , and it was within normal limits. An X-ray film of the chest disclosed marked enlargement of the superior mediastinum (Fig. 1). An ultrasonographic examination disclosed an immobile, unecholucent mass measuring $3.0 \times 4.0 \mathrm{~cm}$ occupying the right atrium. A contrast enhanced computed tomographic (CT) scan of the thorax disclosed a solid, homogenous superior mediastinal mass that filled the lumen of the superior vena cava and the right atrium (Fig. 2). Sagittal view and coronial view on magnetic resonance imaging (MRI) showed the continuous mass from intra superior vena cava to the right atrium (Fig. 3). Superior vena cavograms showed complete blockage of the left inominate vein with multiple collaterals and marked stenosis of the right inominate vein and superior vena cava (Fig. 4).

A biopsy of the right atrial mass was performed via a femoral vein catheter, and the specimen was suspected as being myxoma. The patient was operated on with the presumptive diagnosis of the right atrial myxoma with caval obstruction. A median sternotomy was performed, which revealed a unresectable thymic tumor invading pericardium and superior vena cava. Partial excision of the tumor was performed and the pathology demonstrated thymoma. The right atrium was opened. A large tumor of nodular appearance was found that extended to the superior vena cava. The tumor in the right atrium was resected from the superior vena cava by blunt digital dissection, and was catheterized by Forgaty catheter. Blood flow of superior vena cava was fluent, and the

From the Department of Internal Medicine, *Cardiovascular Surgery, and **Pathology, Hyogo Brain and Heart Center at Himeji, Himeji Received for publication July 30, 1992; Accepted for publication February 16, 1993

Reprint requests should be addressed to Dr. Sachiko Futami, the Department of Internal Medicine, Brain and Heart Center at Himeji, 520 Saisyo kou, Himeji, Hyogo 670, Japan 


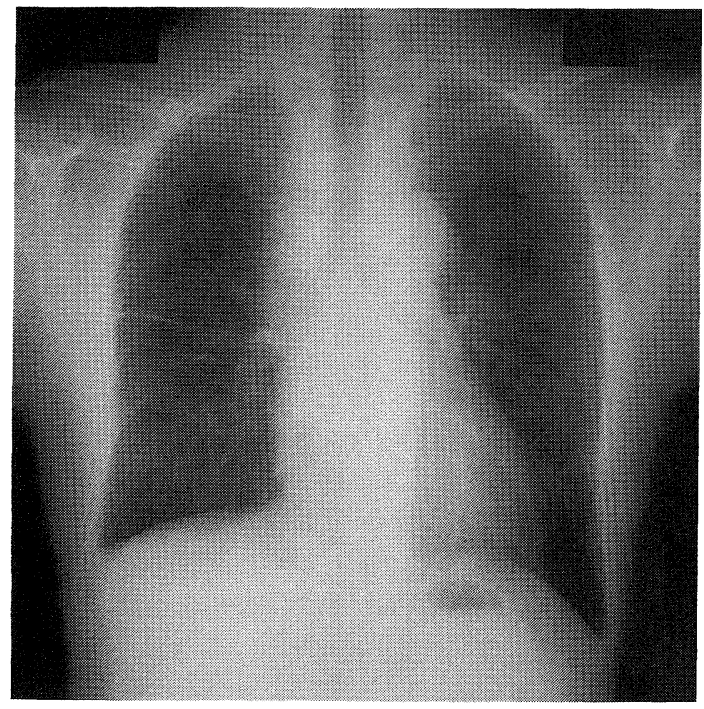

Fig. 1. Radiograph of the chest taken on admission revealing marked enlargement of the superior mediastinum.

operation was finished. Histological examination of the tumor specimen showed mixed type of thymoma with predominant epithelial component. The epithelial cells were growing in a coherent sheet around loosely arranged vascular channels with hyalinized perivascular spaces. Reticulin staining was scanty. The cell nuclei were round to oval and accompanied by occasional nucleolar prominence and high mitotic figures. The cytoplasm were relatively scanty and contained a few diastasesensitive PAS materials. The lymphocytes were distributed in the peripheral zone of the tumor tissue and had no appearance of malignant lymphoma. Epithelial cells

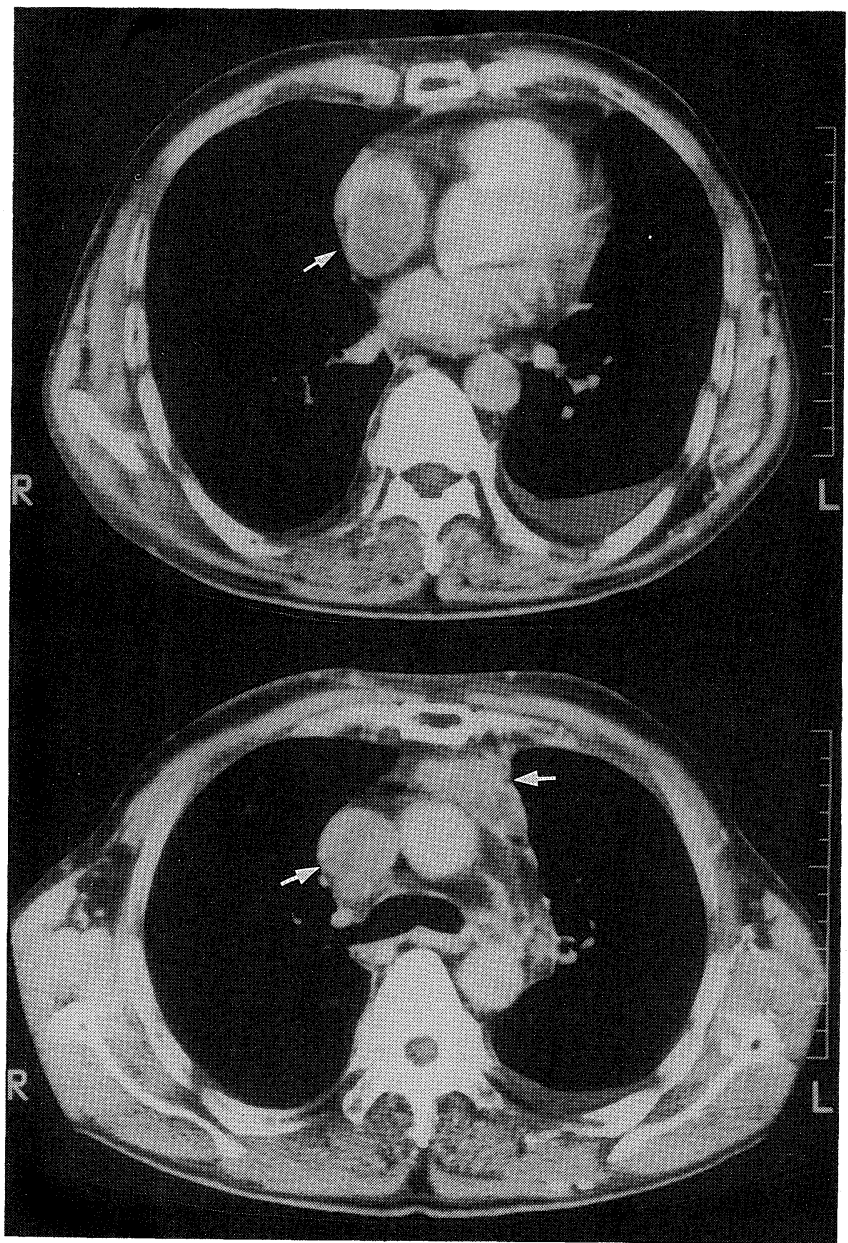

Fig. 2. Contrast enhanced CT of the thorax demonstrating inhomogenous mass in superior vena cava (upper half) and solid, homogenous mass in superior mediastinum (lower half).

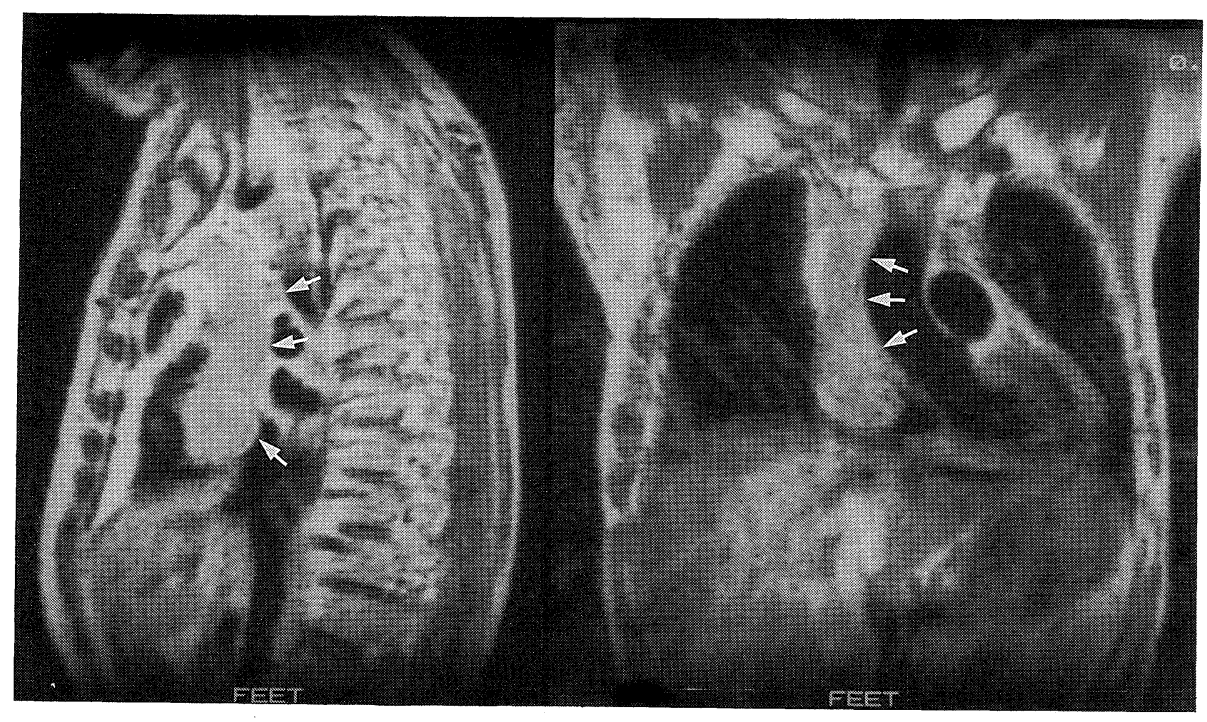

Fig. 3. Sagittal view (left half) and coronal view (right half) on magnetic resonance imaging, disclosing the continuous mass from intra superior vena cava to the right atrium. 


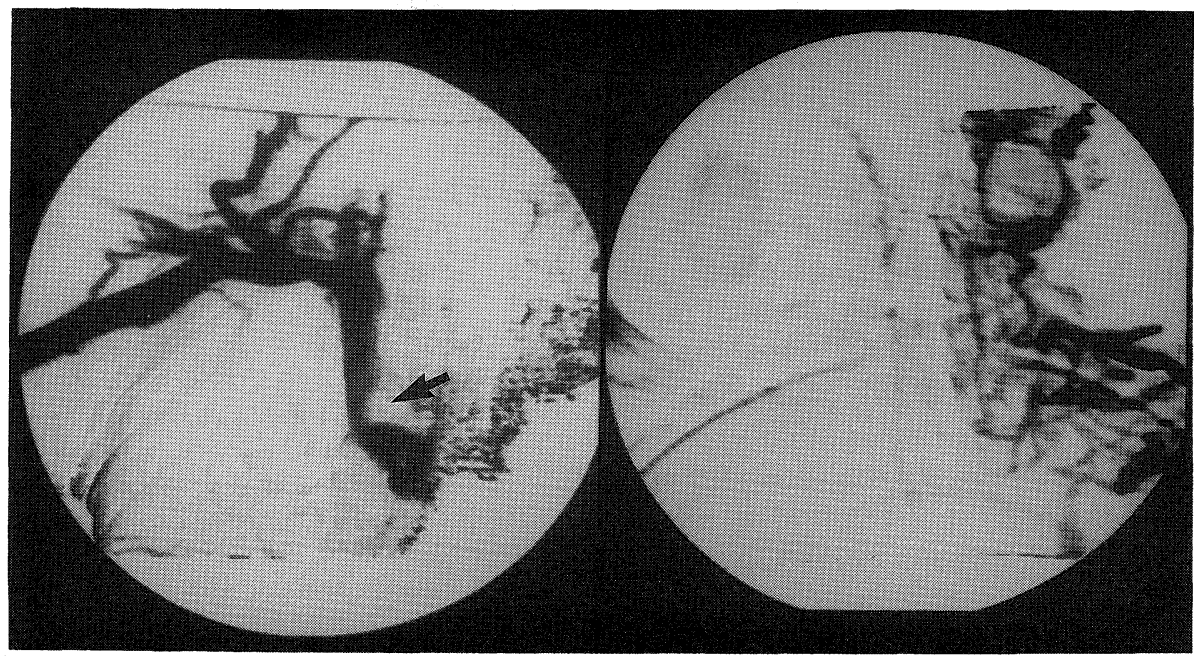

Fig. 4. Superior vena cavograms, disclosing marked stenosis of the right inominate vein (left half) and superior vena cava with multiple collaterals (right half).

showed a positive reaction for EMA, while they were negative for vimentin and desmin. Lymphocytes showed diffuse positive reactions for LCA (lymphocyte common antigen) and UCHL-1, and an occasional reaction to L-26 (Fig. 5).

Postoperatively, the patient recovered uneventfully, and the sign of superior vena cava syndrome disappeared. The patient was sent for radiotherapy.

\section{Discussion}

Approximately half of all thymomas never produce symptoms, and are discovered incidentally, either on a routine chest X-ray or at autopsy. In the present patient, the anterior mediastinal tumor was small, and the symptom was chiefly due to superior vena caval obstruction. Therefore diagnosis was difficult.

Bergh et al reported that superior vena cava syndrome occured in 2 patients among 43 patients with thymomas (1). The presence of superior vena cava synrome has usually been synonymous with obstruction secondary to malignancy. Obstruction of the superior vena cava is essentially due to exentric pressure, invasion of the vein wall by neoplasm or thrombosis $(2-4)$. Tumor extension into the inferior vena cava represents an important and not infrequent complication of abdominal neoplasms. But in the superior vena cava, intravascular extension of neoplasms has rarely been reported. In our review of the literature we were able to find only 16 reports of intracaval and intracardial extension of thymoma $(5-10)$.

When we diagnosed this tumor which had the peculiar invasive form of a thymoma, we undertook various imaging procedures. CT scan provided information concerning the anatomical location, the size and general nature of the anterior mediastinal mass. The intraluminal
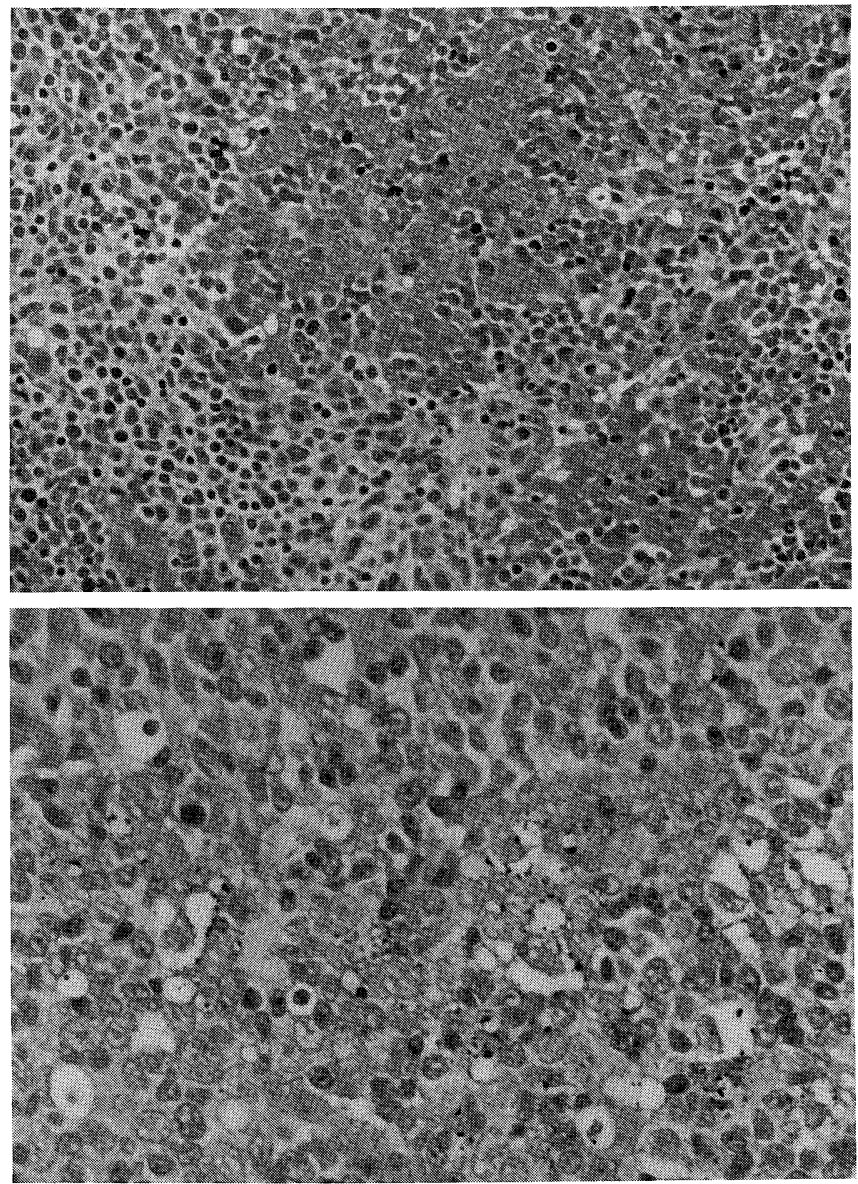

Fig. 5. Mixed type thymoma with epithelial cells and lymphocytes (HE stain, $\times 100$ ) (upper), high power of the left (HE stain, $\times 200$ ) (lower). 
mass was not suggested to be a venous thrombus but rather a tumor thrombus, because the mass was an inhomogenous soft tissue and not water density. MRI provided the continuity of the mass in the superior vena cava and the right atrium. Superior vena cavogram showed the site of obstruction and collateral flow. With the combination of these three studies it was impossible to prove the continuity of the anterior mediastinal mass and intracaval mass. Keen and Libshitz reported that the presence or absence of intervening fat planes on CT accurately predicts vascular and/or pericardial invasion (11). However Fon et al reported that although the presence of preserved fat planes between a thymoma and the adjacent vascular structures reliably excludes macroscopic invasion, the absence of such fat does not consistently indicate an invasive tumor (12). Chiou et al (13) defined three categories of mediastinal fat planes; complete preservation, partial obliteration or preservation, and complete obliteration $(14,15)$. In the present case, CT demonstrated that the tumor had partially obliterated surrounding fat planes, but we could not judge the continuity of the anterior mediastinal mass and intracaval mass with certainty. An unequivocal diagnosis of thymoma can often be made after examination of an open surgical biopsy or even a needle biopsy. In the present case, for the diagnosis of an intracaval mass, histological examination by intravascular catheter was the invasive procedure of choice. The two basic elements of the thymoma glands are the epithelial cells and the lymphocytes. The large majority of the tumors designated as thymomas are composed of a mixture of these two elements, the relative proportions varying considerably from case to case and even in different areas of the same tumor. The designition of thymoma is restricted to neoplasms of the thymic epithelial cells, regardless of the presence or absence of a lymphoid component. It thus follows that identification of epithelial cells constitutes a sine qua non for the histopathologic diagnosis of thymoma. Occasionally, however, differential diagnosis from other tumors, particularly malignant lymphoma and germinoma (seminoma), may be impossible under these circumstances. In some instances, the newly developed immunologic technics may provide a definitive answer. Kodama et al (16) reported that most thymomas are focally or diffusely stained with monoclonal antibody Leu-7, and all thymomas are stained for keratin in varying degrees. This makes a good marker for differential diagnosis between thymoma and lymphoma or seminoma $(16,17)$. We did not perform staining by Leu-7, keratin, but demonstrated the epithelial cells in the tumor reacting to EMA, and the lymphocytes in the tumor had no appearance of malignant lymphoma. Therefore we diagnosed this tumor as thymoma.

Cases of invasive thymomas should be suspected whenever the superior vena cava syndrome is unexpectedly diagnosed, and CT examination is necessary. But if it is not sufficient to simply to predict the character of the tumor, further attempts at establishing the diagnosis are required.

\section{References}

1) Bergh NP, Gatzinsky P, Larsson S, Lundin P, Ridell B. Tumors of the thymus and thymic region; I. Clinicopathological studies on thymomas. Ann Thorac Surg 25: 91, 1978.

2) Gomes MN, Hufuagel CA. Superior vena cava obstruction. A review of the literature and report of 2 cases due to benign intra thoracic tumors. Ann Thorac Surg 20: 344, 1975.

3) Davis RD, Oldham HN, Sabiston DC. Primary cysts and neoplasms of the mediastinum; recent changes in clinical presentation, methods of diagnosis, management, and results. Ann Thorac Surg 44: 229, 1987.

4) Adkins RB, Maples MD, Hainsworth JD. Primary malignant mediastinal tumors. Ann Thorac Surg 38: 648, 1985.

5) Airan B, Sharma R, Iyer KS, Kalia PK, Singh MK. Malignant thymoma presenting as intracardiac tumor and superior vena caval obstruction. Ann Thorac Surg 50: 989, 1990.

6) Tanabe T, Kubo Y, Hashimoto M, Sugie S. Patch angioplasty of the superior vena caval obstruction. J Cardiovasc Surg 20: 519, 1979.

7) Fujio A, Kitano M, Asakura S, et al. Intracaval and intraatrial growth of thymoma. Report of a long surviving case. Nippon Kyobu Shikkan Gakkai Zasshi 23: 934, 1985 (in Japanese).

8) Or R, Raz I, Raveh D, Lichovitzki G, Kleinman Y. Thymoma presenting as a superior vena cava syndrome. Remission following therapy. Klin Wochenschr 65: 617, 1987.

9) Arai T, Inagaki K, Hata E, Hirata E, Onoue Y, Morimoto K. Reconstruction of the superior vena cava in a patient with a thymoma. Chest 73: 230, 1978.

10) Mendez-Fernandez MA, Kremen AF, Greis RC, Henly WS. Reconstruction of the left inominate vein in a patient with invasive thymoma undergoing coronary artery bypass surgery. $\mathbf{J}$ Thorac Cardiovasc Surg 27: 351, 1986.

11) Keen SJ, Libshitz HI. Thymic legions; experience with computed tomography in 24 patients. Cancer 59: 1520, 1987.

12) Fon GT, Bein ME, Mancuso AA, Keesey JC, Lupetin AR, Wong WS. Computed tomography of the anterior mediastinum in myasthesia gravis. A radiographic-pathologic correlative study. Radiology 142: 135, 1982.

13) Chiou GT, Chen CL, Wei J, Hwang WS. Reconstruction of superior vena cava in invasive thymoma. Chest 97: 502, 1990.

14) Bechtold RE, Wolfman NT, Karstaedt N, Choplin RH. Superior vena caval obstruction; detecting using CT. Radiology 157: 485, 1985.

15) Korobkin M, Gasano UA. Intracaval and intracardiac extention of malignant thymoma; CT diagnosis. J Comput Assist Tomogr 13: 348, 1989.

16) Kodama $T$, Watanabe $S$, Sato $Y$, et al. An immunohistochemical study of thymic epithelial tumors. I. Epithelial component. Am J Surg Pathol 10: 26, 1986.

17) Sato $Y$, Watanabe $S$, Mukai $K$, et al. An immunohistochemical study of thymic epithelial tumors. 2. Lymphoid component. Am J Surg Pathol 10: 862, 1986. 